\title{
Atrophy in the Medial Temporal Lobe is Specifically Associated With Encoding and Storage of Verbal Information in $\mathrm{MCl}$ and Alzheimer Patients
}

\author{
Mebeline E. Boon ${ }^{\mathrm{a}}$, Rene J.F. Melis ${ }^{\mathrm{a}}$, Marcel G.M. Olde Rikkert ${ }^{\mathrm{a}}$, \\ Roy P.C. Kessels ${ }^{a, b, c, d}$
}

\begin{abstract}
Background: Alzheimer's dementia (AD) is characterized by a progressive decline in the encoding and storage of episodic memory. Word list learning tests can characterize different aspects of episodic memory. Medial temporal lobe atrophy (MTA) seems to be an important anatomical feature of $\mathrm{AD}$ and its prodromal stage, mild cognitive impairment (MCI). The aim of this study is to define the relationships of the functional memory processes and MTA in patients with $\mathrm{AD}$ and $\mathrm{MCI}$, while correcting for confounding factors.
\end{abstract}

Methods: MTA was evaluated with a visual assessment scale using the MRIs of 53 patients diagnosed with AD or MCI and 19 controls. Rey's Auditory Verbal Learning Test was used to assess the different aspects of memory processing, i.e., encoding, storage and retrieval. Multiple regression analysis was used to investigate the association of MTA with these different memory processes. In addition to general factors such as age, education and sex, white matter lesions, cortical and subcortical atrophy were evaluated for being confounders.

Results: MTA was significantly associated with encoding (corrected beta $=-0.45, \mathrm{sd}=0.11, \mathrm{P}<0.01$ ), and storage (beta $=-0.34$, $\mathrm{sd}=0.12, \mathrm{P}<0.01$ ), but not with retrieval (beta $=-0.18, \mathrm{sd}=0.12$,

Manuscript accepted for publication April 22, 2011

${ }^{a}$ Department of Geriatrics and Alzheimer Center Nijmegen, Radboud University Nijmegen Medical Center, The Netherlands

${ }^{\mathrm{b}}$ Department of Medical Psychology, Radboud University Nijmegen Medical Center, The Netherlands

${ }^{c}$ Donders Institute for Brain, Cognition and Behavior, Radboud University Nijmegen, The Netherlands

${ }^{\mathrm{d}}$ Corresponding author: R.P.C. Kessels, Department of Medical Psychology, Radboud University Nijmegen Medical Center, Internal post 925, Postbus 9101, $6500 \mathrm{HB}$ Nijmegen, The Netherlands. Email: r.kessels@mps.umcn.nl

doi:10.4021/jnr18w
$\mathrm{P}=0.44)$

Conclusions: We can conclude that atrophy of the medial temporal lobe is associated with a decline in encoding and storage of verbal information in MCI and AD.

Keywords: Alzheimer disease; Mild Cognitive Impairment; Episodic memory; Medial temporal lobe; Magnetic resonance imaging; Response bias

\section{Introduction}

Alzheimer's dementia (AD) is a syndrome that is characterized by a progressive decline in multiple cognitive functions. Memory impairment is the key characteristic that is already present in preclinical stages of the disease, often referred to as amnestic mild cognitive impairment (MCI) [1]. Episodic memory, that is the ability to acquire explicit information, is affected most. In contrast, memory of events that occurred earlier in life remains intact for a long time. This reflects deficits in the processes encoding and storage, in contrast to retrieval which is often preserved until late in the disease [2].

There is abundant evidence that the delayed recall score of word-list learning tasks, such as Rey's Auditory Verbal Learning Test (RAVLT), is the best neuropsychological parameter for discriminating $\mathrm{AD}$ patients from healthy controls [2]. This test can also be used to characterize the different functional memory processes. Moreover, the tendency to favor "yes" or "no" responses during a recognition task, the response bias, can be quantified [3]. Although patients with $\mathrm{AD}$ may have a high rate of false alarms, reflecting a liberal response bias, the value of response bias in the differential diagnosis is not clear and data in MCI are lacking altogether.

With the growing availability of magnetic resonance imaging (MRI) in clinical practice, neuroanatomical characteristics of $\mathrm{AD}$ have also been investigated and are increasingly used to support the diagnosis in AD. Atrophy of the medial temporal lobe (MTL) is among the most distinctive neuroanatomical signs. Studies that have investigated the diagnostic value of volumetric and visual assessments of medial 
temporal lobe atrophy (MTA) have indeed proven that these measurements can discern AD patients from normal controls [4]. Next to MTA, general atrophy and white-matter pathology are often present in AD patients.

Although the notion that the MTL is important for memory formation is well established [5], it is less clear whether the specific episodic memory impairment in AD and MCI is directly linked with MTA. Previous studies have studied this relationship and found an association between MTA and memory decline $[6,7]$. However, the influence of covariates such as education level or white-matter pathology has not been taken into account. Furthermore, in these studies memory was considered a unitary function and separate analyses of the different functional processes have not been performed. Next to descriptive analysis of the different MRI and RAVLT variables, including results on response bias, we investigated the neuropsychological correlates of MTA in both MCI and AD patients while correcting for white-matter lesions (WMLs), as well as cortical and subcortical atrophy. Our hypothesis is that storage and encoding specifically rely on the MTL and therefore are inversely associated with MTA in both $\mathrm{AD}$ and MCI patients. Retrieval, on the other hand, will be less associated with the MTA score.

\section{Materials and Methods}

Data of 53 patients were collected from a database of the memory clinic of Radboud University Nijmegen Medical Center for this cross-sectional retrospective study. For patients with the diagnosis of possible or probable AD (NINCDS-ARDRA [8]) or MCI [1], an available MRI scan and an administered RAVLT were included. In addition, data from 19 healthy volunteers who responded to a newspaper advertisement were included. The diagnosis for each patient was made by multidisciplinary consensus. MR imaging examinations were performed using a 1.5 Tesla system (Siemens) and a routine imaging protocol for dementia. All MRI ratings were performed by one rater (MB), who was blind to clinical data. 3D high-resolution T1 weighted coronal slices were used to score the MTA. T2 weighted, fluidattenuated inversion recovery (FLAIR) images were used to assess WMLs, cortical and subcortical atrophy. Linear measurements of subcortical atrophy included the bifrontal ratio, bicaudate ratio and the bioccipital ratio [9]. A measure of cortical atrophy was obtained by dividing the width of the Sylvian fissure by the maximal brain width [9]. White-matter abnormalities were scored according to the scale proposed by Wahlund [10]. The visual rating scale proposed by Scheltens was used to assess MTA [11]. This scale distinguishes between 4 stages of atrophy, which are discerned based on the width of the choroid fissure, width of the temporal horn and height of the hippocampus. All participants performed a brief neuropsychological screening including the Mini Men- tal State Examination (MMSE) and the RAVLT [12]. During the RAVLT, participants had to memorize a list of 15 words, which is presented and tested repeatedly over five learning trials. The participants were asked to recall the words after a delay of 20 minutes (delayed recall), followed by a "yes/no" recognition task of the words from the list intermixed with 15 distracter words. A discrimination index was calculated from the recognition task using the signal detection theory [3]. The C-index was selected to assess the response bias [3].

Linear regression models were used to evaluate the association between the MTA score (left and right averaged) and the memory processes. Memory encoding was quantified with the recall score after the first trial. The storage process was defined as the ability to retain the information stored after the fifth trial over the 20 minutes delay, and was quantified with the discrimination index from the recognition score corrected for the words learned after the fifth trial. The delayed recall task is more dependent on the retrieval process as the recognition task, therefore the retrieval process was quantified with the difference between these two tasks. Age, sex and education level were entered in the model as independent variables. WMLs, cortical and subcortical atrophy were investigated for being confounders, and were entered in the models if they were.

\section{Results}

Table 1 lists the results on the RAVLT and the MR findings for the controls, $\mathrm{AD}$ and $\mathrm{MCI}$ patients. Uncorrected regression analysis showed a significant association between MTAscores and general cognitive function, as measured with the MMSE (beta $=-0.68, \mathrm{sd}=0.08, \mathrm{P}<0.01)$. After correction for storage and encoding processes the association was considerably diminished (beta $=-0.26, \mathrm{sd}=0.10, \mathrm{P}=0.01$ ). With inclusion of the remaining confounders (age, sex, education, subcortical atrophy and WMLs of the left hemisphere) the association remained roughly the same (beta $=-0.24$, $\mathrm{sd}=$ $0.11, \mathrm{P}=0.04)$. The raw association between the storage process and MTA-scores was significant (beta $=-0.26$, $\mathrm{sd}=$ $0.10, \mathrm{P}=0.01$ ). After correcting for confounders (age, sex, education, cortical and subcortical atrophy, WMLs in the left hemisphere), the association increased and remained significant (beta $=-0.34, \mathrm{sd}=0.12, \mathrm{P}<0.01$ ). The raw regression analysis of the encoding process and MTA-scores showed a significant association ( beta $=-0.54, \mathrm{sd}=0.09, \mathrm{P}<0.01$ ). With the addition of the confounding factors (cortical and subcortical atrophy and WML's in the pons) to the equation the association remained significant (beta $=-0.45, \mathrm{sd}=0.11$, $\mathrm{P}<0.01)$. Univariate association of the retrieval process and MTA-scores was small and not significant and remains that way (beta $=-0.18, \mathrm{sd}=0.12, \mathrm{P}=0.44$ ) when corrected for confounders (age, sex, education level, WMLs in the occipital lobe, cortical atrophy). 
Table 1. Results (Means \pm SD or Percentage) on the Word-List Memory Task and MR Findings for the Controls, $\mathrm{MCl}$ Patients and AD Patients

\begin{tabular}{|c|c|c|c|}
\hline & Controls & MCI patients & AD patients \\
\hline $\mathrm{N}$ & 21 & 19 & 34 \\
\hline Age & $76.1(3.8)$ & $72.0(6.8)$ & $77.7(5.8)$ \\
\hline$\%$ Female & $23.8 \%$ & $52.6 \%$ & $61.8 \%$ \\
\hline Education level * & $5.4(1.3)$ & $4.5(1.6)$ & $3.9(1.9)$ \\
\hline $\operatorname{MMSE} \dagger$ & $29.0(1.3)$ & $26.7(2.4)$ & $21.3(3.5)$ \\
\hline \multicolumn{4}{|l|}{ RAVLT +} \\
\hline Trial 1 & $4.8(1.3)$ & $3.7(1.2)$ & $2.2(1.3)$ \\
\hline Trial 2 & $7.7(2.3)$ & $4.4(1.3)$ & $3.4(1.8)$ \\
\hline Trial 3 & $8.8(4.5)$ & $6.0(1.5)$ & $3.7(1.9)$ \\
\hline Trial 4 & $9.8(2.6)$ & $6.2(1.4)$ & $4.1(2.2)$ \\
\hline Trial 5 & $10.7(2.4)$ & $6.1(2.2)$ & $4.1(1.5)$ \\
\hline Delayed recall & $8.7(2.9)$ & $2.7(2.3)$ & $0.6(1.1)$ \\
\hline Delayed recognition & $28.1(1.7)$ & $24.9(2.8)$ & $20.3(3.9)$ \\
\hline Hits & $14.0(0.9)$ & $12.8(2.1)$ & $10.9(2.7)$ \\
\hline Correct rejections & $14.1(1.4)$ & $12.1(2.4)$ & $9.4(4.1)$ \\
\hline Misses & $1.0(0.9)$ & $2.2(2.1)$ & $4.1(2.7)$ \\
\hline False alarms & $0.9(1.4)$ & $2.9(2.4)$ & $5.6(4.1)$ \\
\hline Discrimination index $\S$ & $2.9(0.6)$ & $2.0(0.7)$ & $1.1(0.9)$ \\
\hline Response bias $\mathrm{C}$ index & $0.07(0.3)$ & $-0.07(0.4)$ & $-0.14(0.6)$ \\
\hline MTA ** score & $0.4(0.6)$ & $1.3(0.8)$ & $2.3(0.8)$ \\
\hline WML $\|$ total & $3.8(3.4)$ & $3.4(2.8)$ & $4.8(3.9)$ \\
\hline Sylvian fissure ratio $† \dagger$ & $0.09(0.02)$ & $0.08(0.03)$ & $0.09(0.02)$ \\
\hline Occipital ratio $\$+$ & $0.53(0.03)$ & $0.52(0.04)$ & $0.54(0.04)$ \\
\hline Frontal ratio $\S \S$ & $0.35(0.04)$ & $0.35(0.04)$ & $0.37(0.05)$ \\
\hline Caudal ratio $\boldsymbol{q}$ & $0.16(0.03)$ & $0.17(0.03)$ & $0.18(0.03)$ \\
\hline
\end{tabular}

* Education level: ranges from 0 - 7, higher score indicates higher education [13]. † MMSE: mini mental state examination, ranges from 0 - 30, higher scores are better. ‡ RAVLT: Rey's auditory verbal learning test, trial 1 - trial 5/delayed recall score is the number of words reproduced of the 15 words given. Recognition ranges from $0-30$, a score of 15 is chance and 30 indicated complete recognition [12]. § Discrimination index: difference in standard deviation units between subjects' hit rate and false alarm rate, higher values mean better discrimination [3]. I Response bias: Neutral response bias has a $\mathrm{C}$ value of 0 , conservative bias produces positive $C$ values and liberal response bias results in negative values [3].

** MTA: medial temporal lobe atrophy, ranges from $0-4$, lower scores indicate less atrophy. || WML: white matter lesions, ranges from 0 - 40, lower scores indicate less white matter pathology [10]. †† Sylvian fissure ratio: width of the Sylvian fissure divided

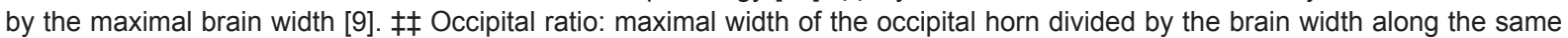
line [9]. §§ Frontal ratio: distance between the tips of the frontal horns divided by the brain width along the same line. TाT Caudal ratio: minimal distance between the caudate indentations of the frontal horns divided by the brain width along the same line [9]. 


\section{Discussion}

Although there is abundant evidence that the MTL is important for episodic memory formation [5], to date it was unclear whether the specific episodic memory impairment in patients with AD pathology is directly associated with MTA. This study showed that, after taking covariates into account, the decline in memory encoding and storage, which is the neuropsychological hallmark of $\mathrm{AD}$ pathology, was most strongly linked to MTA in AD and MCI patients. Initially, the MMSE was also strongly associated with MTA, but the association largely disappeared by correcting for the storage and encoding processes. Secondary, we found a trend towards a more liberal response bias in AD patients as compared to controls and MCI patients, which is in accordance with previous studies [14], but no significant between-group differences were found because of great within-group differences.

The present study extends previous research in that we included both MCI and AD patients and corrected for several MRI parameters, which helped expose the specific association of MTA with encoding and storage of verbal information. Especially cortical atrophy proved to be a confounding factor in the investigation of the association of MTA and cognitive functions. This is understandable as cortical atrophy is associated with a wide range of cognitive deficits. Future research should also correct for these MRI features. In contrast with previous results that have used volumetric measurements of the MTL, we used a visual rating scale. Comparative studies have found good correlations between these two techniques, and even suggest that the visual rating scale is more accurate for the diagnosis of AD [15]. Moreover, it is a scale that is widely used in clinical practice.

We can conclude from this study that specifically encoding and storage impairments in $\mathrm{AD}$ and $\mathrm{MCI}$ patients were directly associated with MTA. This further elucidates the relationship between the neuro-anatomical and neuropsychological features of Alzheimer disease and offers extra legitimacy for MTA measurements as a diagnostic tool for AD pathology. In this study we confirmed that AD patients tend to have a liberal response bias. To further broaden our understanding of the disease mechanisms, it would be interesting to investigate the neuro-anatomical correlates of this liberal response bias.

\section{References}

1. Petersen RC, Smith GE, Waring SC, Ivnik RJ, Tangalos EG, Kokmen E. Mild cognitive impairment: clinical characterization and outcome. Arch Neurol 1999;56(3):303-308.

2. Becker J, Overman A. The memory deficit in Alzheim- er's Disease. In: Baddeley A, Kopelman M, Wilson B, eds. The handbook of memory disorders. John Wiley \& Sons Ltd, 2002;569-589.

3. Snodgrass JG, Corwin J. Pragmatics of measuring recognition memory: applications to dementia and amnesia. J Exp Psychol Gen 1988;117(1):34-50.

4. Laakso MP, Hallikainen M, Hanninen T, Partanen K, Soininen H. Diagnosis of Alzheimer's disease: MRI of the hippocampus vs delayed recall. Neuropsychologia 2000;38(5):579-584.

5. Squire LR, Zola-Morgan S. The medial temporal lobe memory system. Science 1991;253(5026):1380-1386.

6. de Toledo-Morrell L, Dickerson B, Sullivan MP, Spanovic C, Wilson R, Bennett DA. Hemispheric differences in hippocampal volume predict verbal and spatial memory performance in patients with Alzheimer's disease. Hippocampus 2000;10(2):136-142.

7. Petersen RC, Jack CR, Jr., Xu YC, Waring SC, O’Brien PC, Smith GE, Ivnik RJ, et al. Memory and MRI-based hippocampal volumes in aging and AD. Neurology 2000;54(3):581-587.

8. McKhann G, Drachman D, Folstein M, Katzman R, Price D, Stadlan EM. Clinical diagnosis of Alzheimer's disease: report of the NINCDS-ADRDA Work Group under the auspices of Department of Health and Human Services Task Force on Alzheimer's Disease. Neurology 1984;34(7):939-944.

9. Gomori JM, Steiner I, Melamed E, Cooper G. The assessment of changes in brain volume using combined linear measurements. A CT-scan study. Neuroradiology 1984;26(1):21-24.

10. Wahlund LO, Barkhof F, Fazekas F, Bronge L, Augustin M, Sjogren M, Wallin A, et al. A new rating scale for age-related white matter changes applicable to MRI and CT. Stroke 2001;32(6):1318-1322.

11. Scheltens P, Fox N, Barkhof F, De Carli C. Structural magnetic resonance imaging in the practical assessment of dementia: beyond exclusion. Lancet Neurol 2002;1(1):13-21.

12. Rey A. L 'examen clinicique in psychology. Paris: Press universitaire de France, 1964.

13. Verhage F. Intelligentie en leeftijd: onderzoek bij Nederlanders van twaalf tot zevenenzeventig jaar [Intelligence and age: Study with Dutch people from age 12 to 77]. Assen: Van Gorcum, 1964.

14. Kramer JH, Rosen HJ, Du AT, Schuff N, Hollnagel C, Weiner MW, Miller BL, et al. Dissociations in hippocampal and frontal contributions to episodic memory performance. Neuropsychology 2005;19(6):799-805.

15. Wahlund LO, Julin P, Johansson SE, Scheltens P. Visual rating and volumetry of the medial temporal lobe on magnetic resonance imaging in dementia: a comparative study. J Neurol Neurosurg Psychiatry 2000;69(5):630635. 